Research Article

\title{
Jacobian Nonsingularity in Nonlinear Symmetric Conic Programming Problems and Its Application
}

\author{
Yun Wang $(\mathbb{D}$ and Dezhou Kong $\mathbb{D}$ \\ Department of Mathematics and Information Science, School of Information Science and Engineering, \\ Shandong Agricultural University, Tai'an 271000, China \\ Correspondence should be addressed to Dezhou Kong; dezhoukong@163.com
}

Received 22 September 2020; Revised 4 November 2020; Accepted 18 November 2020; Published 31 December 2020

Academic Editor: Xinguang Zhang

Copyright (C) 2020 Yun Wang and Dezhou Kong. This is an open access article distributed under the Creative Commons Attribution License, which permits unrestricted use, distribution, and reproduction in any medium, provided the original work is properly cited.

\begin{abstract}
This paper considers the nonlinear symmetric conic programming (NSCP) problems. Firstly, a type of strong sufficient optimality condition for NSCP problems in terms of a linear-quadratic term is introduced. Then, a sufficient condition of the nonsingularity of Clarke's generalized Jacobian of the Karush-Kuhn-Tucker (KKT) system is demonstrated. At last, as an application, this property is used to obtain the local convergence properties of a sequential quadratic programming- (SQP-) type method.
\end{abstract}

\section{Introduction}

In this paper, we consider the nonlinear symmetric conic programming (NSCP) as follows:

$$
\begin{aligned}
& \min _{x \in X} f(x), \\
& \text { s.t. } h(x)=0, \\
& g(x) \in K,
\end{aligned}
$$

where $X$ and $Y$ are two finite dimensional real vector spaces; $f: X \longrightarrow \Re, h: X \longrightarrow \Re^{m}$, and $g: X \longrightarrow Y$ are twice continuously differentiable functions; and $K \in Y$ is a symmetric cone defined by Euclidean Jordan algebras. In the following part, unless otherwise specified, we denote $X, Y$, and $Z$ to represent finite dimensional real vector spaces with a scalar product $\langle\cdot, \cdot\rangle$ and norm $\|\cdot\|$.

It is well-known that the Karush-Kuhn-Tucker (KKT) conditions of optimization problem (1) are equivalent to the KKT system, which is a nonsmooth system with the metric projector over the symmetric cone. The nonsingularity of Clarke's generalized Jacobian of the KKT system introduced by Pang and Qi [1] is not only one of the most important concepts in perturbation analysis of optimization problems but also plays a vital part in the design of the algorithms and the analysis of the convergence [2-4].

When $K$ in problem (1) is a polyhedral set, Robinson [5] has showed that the strong second-order sufficient condition and the LICQ imply the nonsingularity of Clarke's generalized Jacobian of the KKT system. Interestingly, the converse is also true $[2,6,7]$. Bonnans and Ramírez [8] and Sun [9] demonstrate the equivalent conditions to the nonsingularity of the second-order cone programming problem (SOCP) and the semidefinite programming problem (SDP), respectively.

When $K$ is the class of $C^{2}$-cone reducible sets ([3], Definition 3.135), there are lots of most important results about the Aubin property and the robust isolated calmness of the KKT solution mapping, which guarantee the nonsingularity of the KKT system (see [10-13] and the references therein).

For symmetric cone programming problem, Kong, Tunçel, and Xiu [14-16] use a triangular representation of the Jacobian of Löwner operator to characterize the structure of Clarke's generalized Jacobian of metric projection operator onto symmetric cone. They consider the linear symmetric cone programming problem as follows: 


$$
\begin{aligned}
& \min \langle c, x\rangle, \\
& \text { s.t. } A(x)=b, x \in K,
\end{aligned}
$$

and present five equivalent conditions to the nonsingularity of Clarke's generalized Jacobian of KKT system in [15].

In this paper, we focus on the nonsingularity of Clarke's generalized Jacobian of the KKT system in the setting of the nonlinear symmetric cone programming problem (1). In order to present the optimality conditions of NSCP, we need the variational analysis of symmetric cones and some important sets such as tangent cone. We found that, almost at the same time, we [17] and Kong et al. [15] independently obtained the same expressions of the tangent cone and so on by different approaches (see Proposition 2). Importantly, we introduce a linear-quadratic function to establish the second-order optimality conditions. Using the Euclidean Jordan Algebras and the Peirce decomposition of a finitedimensional vector space, we obtain an upper bound of the linear-quadratic function. Under the constraint nondegeneracy condition, we demonstrate our main result that if a kind of strong second-order sufficient condition holds, any element in Clarke's generalized Jacobian of the KKT system is nonsingular.

In [18], the local convergence for an SQP-type method is ensured by the nonsingularity of Clarke's generalized Jacobian. In this paper, as an application, we give an SQP-type method to solve NSCP (1). The analysis of the local convergence is presented by using the above properties of the nonsingularity, and our proof is a natural extension of the nonlinear programming problem.

The paper is organized as follows. In Section 2, it gives some preliminaries which are used in the paper, including the fundamental notations in Euclidean Jordan algebras. The properties of a linear-quadratic function are developed. In Section 3, we describe the KKT condition and a kind of second-order sufficient condition of NSCP (1) using the linear-quadratic function. Then, we discuss the nonsingularity of Clarke's generalized Jacobian of the equation reformulation of the KKT system. Lastly, the local convergence of a SQP-type method is analyzed by using the nonsingularity in Section 4.

\section{Preliminaries}

In this section, some preliminaries used in the paper are given firstly. Then, we introduce the decomposition results in Euclidean Jordan algebras, which are vital to this paper.

For a locally Lipschitz continuous function $\Xi: \mathscr{O} \subseteq Y \longrightarrow Z$, Clarke's generalized Jacobian of $\Xi$ at $y$ is defined by

$$
\partial \Xi(y):=\operatorname{conv}\left\{V: V=\lim _{k \longrightarrow \infty} \Xi^{\prime}\left(y^{k}\right), y^{k} \in \mathcal{O}_{\Xi}, y^{k} \longrightarrow y\right\},
$$

where $\mathcal{O}_{\Xi}$ is the set of F-differentiable points in $\mathcal{O}$ and "conv" denotes the convex hull.

The next conclusion of the chain rule is given in [19], which is stronger than its first version in [9].
Lemma 1. Suppose that $\Psi: X \longrightarrow Y$ is a continuously differentiable function and $\Xi: O \subseteq Y \longrightarrow Z$ is a locally Lipschitz continuous function. Denote $y^{*}:=\Psi\left(x^{*}\right) \in \mathcal{O}$ and let $\Psi^{\prime}\left(x^{*}\right): X \longrightarrow Y$ be onto. Then, the composite function $\Phi(x):=\Xi(\Psi(x))$ is F-differentiable at $x \in \widehat{N}$ if and only if $\Xi$ is F-differentiable at $\Psi(x)$, where $\hat{N}$ is an open neighborhood of $x^{*}$ and

$$
\partial_{B} \Phi\left(x^{*}\right)=\partial_{B} \Xi\left(y^{*}\right) \Psi^{\prime}\left(x^{*}\right) .
$$

The following conclusion of implicit functions can be obtained from [20] (Section 7.1) and [21] (Lemma 2) directly.

Lemma 2. Let $\Phi: X \times Y \longrightarrow X$ be a locally Lipschitz continuous function and $\Phi(\bar{x}, t \bar{y})=0$. Suppose that any element in $\Pi_{X} \partial \Phi(\bar{x}, t \bar{y})$ is nonsingular. Then, there exists a locally Lipschitz continuous function $x(\cdot): \mathscr{O}_{Y} \longrightarrow X$ satisfying $x(\bar{y})=\bar{x}$ and

$$
\Phi(x(y), y)=0,
$$

where $\mathcal{O}_{Y}$ is an open neighborhood of $\bar{y}$. Furthermore, if $\Phi$ is (strongly) semismooth, then $x(\cdot)$ is (strongly) semismooth.

In the last part of this section, we provide some properties about the metric projector over a convex set $C$ in Banach space (see [22]).

Lemma 3. Suppose that $C$ is a convex set in a Banach space $Z$. Then, for any $y \in Z$ and $V \in \partial \Pi_{C}(y), V$ is self-adjoint. Furthermore, for any $d \in Z, \quad\langle d, V d\rangle \geq 0$ and $\langle V d, d-V d\rangle \geq 0$.

2.1. Euclidean Jordan Algebras. In this part, we show some useful notations and conclusions on Euclidean Jordan Algebras introduced in [23]. Suppose that $\mathbb{F}$ is the real field $\mathbb{R}$ and $\mathbb{V}$ is a finite-dimensional vector space over $\mathbb{F}$.

For any $x \in \mathbb{V}$, denote

$$
\mathscr{L}(x) y:=x \cdot y \text { for every } y \in \mathbb{V} \text {. }
$$

The pair $\mathbb{A}:=(\mathbb{V}, \cdot)$ defined over the real field $\mathbb{R}$ is called a Euclidean Jordan algebra, if, for all $x, y \in \mathbb{V}$ :

(i) $x \cdot y=y \cdot x$

(ii) $x \cdot\left(x^{2} \cdot y\right)=x^{2} \cdot(x \cdot y)$, where $x^{2}:=x \cdot x$

(iii) $\langle x \cdot y, z\rangle_{\mathbb{V}}=\langle y, x \cdot z\rangle_{\mathbb{V}}$

Here are some common concepts used in this paper.

An element $c \in \mathbb{V}$ is called to be the unit element of $\mathbb{A}$ if $x \cdot e=e \cdot x=x$ for all $x \in \mathbb{V}$. We always assume that there exists a unit element $e \in \mathbb{V}$ of $\mathbb{A}=(\mathbb{V}, \cdot)$ in the following paper.

If is called to be idempotent. If two idempotents $p$ and $q$ satisfy $p \cdot q=0$, they are called orthogonal. And $k$ orthogonal idempotents $\left\{c_{1}, c_{2}, \ldots, c_{k}\right\}$ are said to be a complete system if they satisfy 


$$
\sum_{j=1}^{k} c_{j}=e
$$

If a nonzero idempotent $q$ cannot be written as the sum of two other nonzero idempotents, we say $q$ is primitive. And Jordan frame is a complete system of orthogonal primitive idempotents.

The following theorem in [23] is very important to show the spectral decomposition.

Theorem 1. Let $\mathbb{A}=(\mathbb{V}, \cdot)$ be a Euclidean Jordan algebra and $R(\mathbb{A})=r$. Then, for any $x \in \mathbb{V}$,

$$
x=\sum_{j=1}^{r} \lambda_{j}(x) c_{j}=\lambda_{1}(x) c_{1}+\lambda_{2}(x) c_{2}+\cdots+\lambda_{r}(x) c_{r},
$$

where $\left\{c_{1}, c_{2}, \ldots, c_{r}\right\}$ is a Jordan frame and $\lambda_{j}(x) \in \mathbb{R}, j=1, \ldots, r, \quad$ satisfying $\quad \lambda_{1}(x) \geq \lambda_{2}(x) \geq \cdots \geq$ $\lambda_{r}(x)$.

We say the numbers $\lambda_{1}(x), \lambda_{2}(x), \ldots, \lambda_{r}(x)$ to be eigenvalues of $x$. Then, $x$ has the spectral decomposition (8) and

$$
\operatorname{tr}(x)=\sum_{j=1}^{r} \lambda_{j}(x)
$$

Therefore, another associative inner product can be defined by using $\operatorname{tr}(x):\langle x, y\rangle:=\operatorname{tr}(x \cdot y), x, y \in \mathbb{V}$. Let $\|\cdot\|$ be the norm on $\mathbb{V}$ induced by this inner product, then

$$
\|x\|:=\sqrt{\langle x, x\rangle}=\sqrt{\sum_{j=1}^{r} \lambda_{j}^{2}(x), \quad x \in \mathbb{V} .}
$$

For a scalar-valued function $\phi: \mathbb{R} \longrightarrow \mathbb{R}$, we define Löwner's operator associated with $\mathbb{A}=(\mathbb{V}, \cdot)$ from $[24]$ as

$$
\begin{aligned}
\phi_{\mathbb{V}}(x):= & \sum_{j=1}^{r} \phi\left(\lambda_{j}(x)\right) c_{j}=\phi\left(\lambda_{1}(x)\right) c_{1}+\phi\left(\lambda_{2}(x)\right) c_{2} \\
& +\cdots+\phi\left(\lambda_{r}(x)\right) c_{r},
\end{aligned}
$$

where $x=\sum_{j=1}^{r} \lambda_{j}(x) c_{j} \in \mathbb{V}$.

The metric projection operator on

$$
K:=\left\{y^{2}: y \in \mathbb{V}\right\}
$$

can be described by Löwner's operator using $\phi(t)=\max (0, t)$ as follows:

$$
\Pi_{K}(x)=x_{+}=\left(\lambda_{1}(x)\right)_{+} c_{1}+\left(\lambda_{2}(x)\right)_{+} c_{2}+\cdots+\left(\lambda_{r}(x)\right)_{+} c_{r},
$$

which is very important in the following research.

It is known from ([23], Theorem III.2.1) that the above cone $K=\left\{y^{2}: y \in \mathbb{V}\right\}$ is a self-dual homogeneous closed convex cone, we call it a symmetric cone, which is the constraint set of problem (1)
For a Jordan frame of $\mathbb{A}\left\{c_{1}, c_{2}, \ldots, c_{r}\right\}$, we denote $\mathbb{V}_{i j}$ as follows:

$$
\mathbb{V}_{i j}:= \begin{cases}\mathbb{V}\left(c_{i}, 1\right), & i=j, \\ \mathbb{V}\left(c_{i}, \frac{1}{2}\right) \cap \mathbb{V}\left(c_{j}, \frac{1}{2}\right), & i \neq j .\end{cases}
$$

Suppose that $\mathscr{C}_{i j}(x)$ is the orthogonal projection onto $\mathbb{V}_{i j}$. Then, $\mathscr{C}_{i j}(x)$ has the following expression:

$$
\mathscr{C}_{j l}(x)=\sum_{i=1}^{d}\left\langle v_{j l}^{(i)}, \cdot\right\rangle v_{j l}^{(i)}
$$

where $\left\{v_{j l}^{(i)}\right\}_{i=1}^{d} \in \mathbb{V}$ denotes orthonormal vectors and $d$ satisfies $n=r+d / 2 r(r-1)$.

It follows from [25] that

$$
\begin{aligned}
h= & \sum_{j=1}^{r} \mathscr{C}_{j j}(x) h+\sum_{1 \leq j<l \leq r} \mathscr{C}_{j l}(x) h=\sum_{j=1}^{r}\left\langle c_{j}, h\right\rangle c_{j} \\
& +\sum_{1 \leq j<l \leq r} 4 c_{j} \cdot\left(c_{l} \cdot h\right), \quad \forall h \in \mathbb{V} .
\end{aligned}
$$

Actually, all the eigenvectors

$$
\left\{c_{1}, c_{2}, \ldots, c_{r}, v_{j l}^{(1)}, v_{j l}^{(2)}, \ldots, v_{j l}^{(d)}, \quad 1 \leq j<l \leq r\right\}
$$

form an orthonormal basis of $\mathbb{V}$.

Assume that there exist two integers $s$ and $s_{1}$ such that

$$
\begin{aligned}
\lambda_{1}(x) & \geq \cdots \geq \lambda_{s}(x)>0=\lambda_{s+1}(x)=\cdots=\lambda_{s_{1}}(x)>\lambda_{s_{1}+1}(x) \\
& \geq \cdots \geq \lambda_{r}(x) .
\end{aligned}
$$

Let us introduce three index sets:

$$
\begin{aligned}
& \alpha:=\{1, \ldots, s\}, \\
& \beta:=\left\{s+1, \ldots, s_{1}\right\}, \text { and } \gamma:=\left\{s_{1}+1, \ldots, r\right\} .
\end{aligned}
$$

For $1 \leq j \leq l$, denote

$$
\begin{aligned}
& h_{j j}:=\mathscr{C}_{j j}(x) h=\left\langle c_{j}, h\right\rangle c_{j}, \\
& h_{j l}:=\mathscr{C}_{j l}(x) h=\sum_{i=1}^{d}\left\langle v_{j l}^{(i)}, h\right\rangle v_{j l}^{(i)}, \\
& h_{\alpha \alpha}=\sum_{j=1}^{s} h_{j j}+\sum_{1 \leq j<l \leq s} h_{j l}, \\
& h_{\alpha \beta}=\sum_{j=1}^{s} \sum_{l=s+1}^{s_{1}} h_{j l}, \\
& h_{\alpha \gamma}=\sum_{j=1}^{s} \sum_{l=s_{1}+1}^{r} h_{j l}, \\
& h_{\beta \beta}=\sum_{j=s+1}^{s_{1}} h_{j j}+\sum_{s+1 \leq j<l \leq s_{1}} h_{j l}, \\
& h_{\beta \gamma}=\sum_{j=s+1}^{s_{1}} \sum_{l=s_{1}+1}^{r} h_{j l}, \quad \sum_{j=s_{1}+1}^{r} h_{j j}+\sum_{s_{1}+1 \leq j<l \leq r} h_{j l} .
\end{aligned}
$$


For the merit projector $\Pi_{K}$ onto the symmetric cone, Sun and Sun [25] has proved that $\Pi_{K}$ is strongly semismooth everywhere. Wang [17] gave a characterization pertaining the structure of the B-subdifferential of $\Pi_{K}$. Recently, Kong, Tunçel, and Xiu presented an exact expression for B-subdifferential and Clarke's generalized Jacobian of $\Pi_{K}[14,15]$. Here, we rewrite the conclusion obtained by Kong et al. [15] as follows.

Proposition 1. Let $x=\sum_{j=1}^{r} \lambda_{j}(x) c_{j}$ and the indexsets $\alpha, \beta, \gamma$ be given by (19). Then, for any $V \in \partial \Pi_{K}(x)$, there exists $W \in \partial \Pi_{K^{|\beta|}}(0)$ such that

$$
V h=\sum_{j=1}^{s} h_{j j}+\sum_{j=1}^{s} \sum_{l=j+1}^{s_{1}} h_{j l}+\sum_{j=1}^{s} \sum_{l=s_{1}+1}^{r} \frac{\lambda_{j}(x)}{\lambda_{j}(x)-\lambda_{l}(x)} h_{j l}+W h,
$$

where $K^{|\beta|}$ is the symmetric cone in subspace $\mathbb{V}_{\beta \beta}:=\oplus_{i \leq j, i \in \beta, j \in \beta} \mathbb{V}_{i j}$.

In order to describe the optimality conditions of NSCP (1), we need the expressions of some important sets, such as the tangent cone of $\mathrm{K}$ at $x_{+}\left(\mathscr{T}_{K}\left(x_{+}\right)\right)$, the linearity of $\mathscr{T}_{K}\left(x_{+}\right)\left(\operatorname{lin}\left(\mathscr{T}_{K}\left(x_{+}\right)\right)\right)$, critical cone $C\left(x_{+}\right)$, and the affine space of $C\left(x_{+}\right)$, denoted by aff $\left(C\left(x_{+}\right)\right)$. As Wang [17] and Kong et al. [15] have obtained the characterizations independently, we only show the topological results in the following proposition.

Proposition 2. Let $x$ have eigenvalues as in (18). It holds that

$$
\begin{aligned}
\mathscr{T}_{K}\left(x_{+}\right) & =\left\{z: h_{\beta \beta}+h_{\beta \gamma}+h_{\gamma \gamma} \geqslant 0\right\}, \\
\operatorname{lin}\left(\mathscr{T}_{K}\left(x_{+}\right)\right) & =\left\{h: h_{\beta \beta}=0, h_{\beta \gamma}=0, h_{\gamma \gamma}=0\right\}, \\
C\left(x_{+}\right) & =\left\{h: h_{\beta \beta} \geqslant 0, h_{\beta \gamma}=0, h_{\gamma \gamma}=0\right\}, \\
\operatorname{aff}\left(C\left(x_{+}\right)\right) & =\left\{h: h_{\beta \gamma}=0, h_{\gamma \gamma}=0\right\} .
\end{aligned}
$$

2.2. A Linear-Quadratic Function. Inspired by the works by Bonnans and Shapiro [2] and Sun [9], we define a linearquadratic function as follows, which is vital for establishing a kind of strong second-order sufficient condition.

Definition 1. For any given $v \in \mathbb{V}$, define a linear-quadratic function $\Upsilon_{v}: \mathbb{V} \times \mathbb{V} \longrightarrow \mathfrak{R}$ by

$$
\begin{aligned}
\Upsilon_{v}(z, h) & :=4\left\langle z \cdot h, v^{\dagger} \cdot h\right\rangle, \\
(z, h) & \in \mathbb{V} \times \mathbb{V},
\end{aligned}
$$

where $v^{\dagger}$ is the Moore-Penrose pseudoinverse of $v$.

Here, the linear-quadratic function is different from Definition 3.2 in [15]. Even in the especial case, $v \in K$ and $v \cdot z=0$, the value of the linear-quadratic function (26) is twice the one in [15].

For the linear-quadratic function (16), we have the following conclusions.

Proposition 3. If $h \in$ aff $C\left(x_{+}\right)$, then

$$
\Upsilon_{x_{+}}\left(x-x_{+}, h\right)=\sum_{j=1}^{s} \sum_{l=s_{1}+1}^{r} \frac{\lambda_{l}}{\lambda_{j}}\left\|h_{j l}\right\|^{2} .
$$

Proof. As $x_{+}=\sum_{j=1}^{s} \lambda_{j} c_{j}, x-x_{+}=\sum_{j=s_{1}+1}^{r} \lambda_{j} c_{j}$, we have

$$
\begin{aligned}
\left(x-x_{+}\right) \cdot h & =\sum_{j=1}^{s} \sum_{l=s_{1}+1}^{r} \sum_{i=1}^{d} \lambda_{l}\left\langle v_{j l}^{(i)}, h\right\rangle\left(c_{l} \cdot v_{j l}^{(i)}\right), \frac{n !}{r !(n-r) !} \\
\left(x_{+}\right)^{\dagger} \cdot h & =\sum_{j=1}^{s} \lambda_{j}^{-1}\left\langle c_{j}, h\right\rangle c_{j}+\sum_{j=1}^{s} \sum_{l=s_{1}+1}^{r} \sum_{i=1}^{d} \lambda_{j}^{-1}\left\langle v_{j l}^{(i)}, h\right\rangle\left(c_{j} \cdot v_{j l}^{(i)}\right),
\end{aligned}
$$

and (28) comes from the definition of $\Upsilon_{x_{+}}\left(x-x_{+}, h\right)$.

Lemma 4. Let $b \in K$ and $-r \in \mathcal{N}_{K}(b)$. Then, for any $v \in \partial \Pi_{K}(b-r)$,

$$
\langle\Delta b, \Delta r\rangle \geq \Upsilon_{b}(r, \Delta b)
$$

where $\Delta b, \Delta r \in \mathbb{V}$ satisfying $\Delta b=v(\Delta b+\Delta r)$.

Proof. Denote $x:=b-r$. Then,

$b=\Pi_{K}(b-r)=\Pi_{K}(x)$ and $b \cdot r=r \cdot b=0, \quad b \in K, r \in K$.

Thus, we assume that $x$ has the following spectral decomposition:

$$
x=\sum_{j=1}^{s} \lambda_{j}(x) c_{j}+\sum_{j=s_{1}+1}^{r} \lambda_{j}(x) c_{j}
$$

satisfying (18), and $b$ and $r$ have the spectral decompositions as follows:

$$
\begin{aligned}
& b=\sum_{j=1}^{s} \lambda_{j}(x) c_{j}, \\
& r=\sum_{j=s_{1}+1}^{r}\left(-\lambda_{j}(x)\right) c_{j} .
\end{aligned}
$$

From Proposition 1, we have, for any $V \in \partial \Pi_{K}(x)$, there exists $W \in \partial \Pi_{K^{|\beta|}}(0)$ such that

$$
V h=\sum_{j=1}^{s} h_{j j}+\sum_{j=1}^{s} \sum_{l=j+1}^{s_{1}} h_{j l}+\sum_{j=1}^{s} \sum_{l=s_{1}+1}^{r} \frac{\lambda_{j}(x)}{\lambda_{j}(x)-\lambda_{l}(x)} h_{j l}+W h .
$$

Therefore, we have from $\Delta b=v(\Delta b+\Delta r)$ that 


$$
\begin{aligned}
& \sum_{j=1}^{s}\left[(\Delta b)_{j j}+(\Delta r)_{j j}\right]+W(\Delta b+\Delta r) \\
& \quad+\sum_{j=1}^{s} \sum_{l=j+1}^{s_{1}}\left[(\Delta b)_{j l}+(\Delta r)_{j l}\right]+\sum_{j=1}^{s} \sum_{l=s_{1}+1}^{s} \frac{\lambda_{j}(x)}{\lambda_{j}(x)-\lambda_{l}(x)} \\
& \quad \cdot\left[(\Delta b)_{j l}+(\Delta r)_{j l}\right] \\
& =\sum_{j=1}^{s}(\Delta b)_{j j}+\sum_{j=s_{1}}^{r}(\Delta b)_{j j}+(\Delta b)_{\beta \beta}+(\Delta b)_{\alpha \gamma}+(\Delta b)_{\beta \gamma}+(\Delta b)_{\gamma \gamma},
\end{aligned}
$$

which implies

$$
\begin{gathered}
\sum_{j=1}^{s}(\Delta r)_{j j}=0 \\
\sum_{j=s_{1}+1}^{r}(\Delta b)_{j j}=0 \\
\sum_{j=1}^{s} \sum_{l=j+1}^{s_{1}}(\Delta r)_{j l}=0 \\
\sum_{l=s_{1}+1}^{r} \sum_{j=s+1}^{l-1}(\Delta b)_{j l}=0, \\
\sum_{j=1}^{s} \sum_{l=s_{1}+1}^{r} \frac{\lambda_{j}(x) \quad(x)-\lambda_{l}(x)}{\left.\lambda_{j}(\Delta r)_{j l}+(\Delta b)_{j l}\right]}=\sum_{j=1}^{s} \sum_{l=s_{1}+1}^{r}(\Delta b)_{j l}, \\
W(\Delta b+\Delta r)=(\Delta b)_{\beta \beta} .
\end{gathered}
$$

We can easily check that

$$
W(\Delta b+\Delta r)=W\left((\Delta b)_{\beta \beta}+(\Delta r)_{\beta \beta}\right) \text {. }
$$

Then, by the properties of the projection of the metric projector in Lemma 3,

$$
\begin{aligned}
\left\langle(\Delta b)_{\beta \beta},(\Delta r)_{\beta \beta}\right\rangle= & \left\langle W(\Delta b+\Delta r),(\Delta b)_{\beta \beta}\right. \\
& \left.+(\Delta r)_{\beta \beta}-W(\Delta b+\Delta r)\right\rangle \\
= & \left\langle(\Delta b)_{\beta \beta}+(\Delta r)_{\beta \beta},\left(W-W^{2}\right)\right. \\
& \left.\cdot\left((\Delta b)_{\beta \beta}+(\Delta r)_{\beta \beta}\right)\right\rangle \geq 0 .
\end{aligned}
$$

Hence, by equations (35)-(37), we obtain from $b^{\dagger}=\sum_{j=1}^{r} \lambda_{j}^{-1}(x) c_{j}$ that

$$
\begin{aligned}
\langle\Delta b, \Delta r\rangle= & \left\langle\sum_{j=1}^{s}(\Delta b)_{j j}+\sum_{j=1}^{s} \sum_{l=j+1}^{r}(\Delta b)_{j l}+\Delta b_{\beta \beta}, \sum_{j=s_{1}+1}^{r}(\Delta r)_{j j}\right. \\
& \left.+\sum_{l=s_{1}+1}^{r} \sum_{j=1}^{l-1}(\Delta r)_{j l}+\Delta r_{\beta \beta}\right\rangle \\
\geq & \left\langle\sum_{j=1}^{s} \sum_{l=s_{1}+1}^{r}(\Delta b)_{j l}, \sum_{j=1}^{s} \sum_{l=s_{1}+1}^{r}(\Delta r)_{j l}\right\rangle \\
= & -\sum_{j=1}^{s} \sum_{l=s_{1}+1}^{r} \frac{\lambda_{l}(x)}{\lambda_{j}(x)}\left\|(\Delta b)_{j l}\right\|^{2}=4\left\langle r \cdot \Delta b, b^{\dagger} \cdot \Delta b\right\rangle \\
= & \Upsilon_{b}(r, \Delta b) .
\end{aligned}
$$

The proof is completed.

\section{Optimality Conditions and Nonsingularity}

We consider nonlinear conic problem (1). Let $x$ belong to the feasible set of problem (1). If

$$
0 \in \operatorname{int}\left\{\left(\begin{array}{l}
h(x) \\
g(x)
\end{array}\right)+\left(\begin{array}{l}
h^{\prime}(x) \\
g^{\prime}(x)
\end{array}\right) X-\left(\begin{array}{l}
0 \\
K
\end{array}\right)\right\},
$$

we say that Robinson's constraint qualification holds at $x$. Then, there exists a Lagrange multiplier $(y, z) \in \mathfrak{R}^{m} \times Y$ satisfying the following KKT conditions:

$$
\begin{aligned}
\nabla_{x} L(x, y, z) & =0, \\
h(x) & =0, \quad-z \in \mathcal{N}_{K}(g(x)),
\end{aligned}
$$

where

$$
L(x, y, z)=f(x)+\langle y, h(x)\rangle-\langle z, g(x)\rangle
$$

is the Lagrangian function of (1). Let $\Lambda(x) \subset \mathfrak{R}^{m} \times Y$ be the set of all the Lagrangian multipliers.

It is easy to verify the KKT conditions (42) which can be equivalently expressed as

$$
\begin{aligned}
F(x, y, z) & =\left(\begin{array}{c}
\nabla_{x} L(x, y, z) \\
h(x) \\
g(x)-\Pi_{K}(g(x)-z)
\end{array}\right) \\
& =\left(\begin{array}{c}
\nabla_{x} L(x, y, z) \\
h(x) \\
z-\Pi_{K}(z-g(x))
\end{array}\right)=0 .
\end{aligned}
$$


Step 0: for a given initial point $\left(x^{1}, y^{1}, z^{1}\right)$, calculate the value of $h\left(x^{1}\right), g\left(x^{1}\right), \nabla f\left(x^{1}\right), h^{\prime}\left(x^{1}\right)$ and $g^{\prime}\left(x^{1}\right)$. Set $k:=1$. Step 1: if $\nabla_{x} L\left(x^{k}, y^{k}, z^{k}\right)=0, h\left(x^{k}\right)=0, g\left(x^{k}\right) \in Q$, stop.

Step 2: calculate $M_{k}=M\left(x^{k}, y^{k}, z^{k}\right)$ and find a KKT point $\left(\Delta x^{k}, y_{\mathrm{QP}}^{k}, z_{\mathrm{QP}}^{k}\right)$ of $\left(\mathrm{Sub}_{k}\right)$ by solving the KKT system (67). Step 3: set $x^{k+1}:=x^{k}+\Delta x^{k}, y^{k+1}:=y_{\mathrm{QP}}^{k}, z^{k+1}:=z_{\mathrm{QP}}^{k}$.

Step 4: calculate $h\left(x^{k+1}\right), g\left(x^{k+1}\right), \nabla f\left(x^{k+1}\right), h^{\prime}\left(x^{k+1}\right)$ and $g^{\prime}\left(x^{k+1}\right)$. Set $k:=k+1$. Go to step 1 .

Algorithm 1: Local SQP method.

For a general constraint $G(x) \in K^{\prime}, x \in X$, where $G: X \longrightarrow Y^{\prime}$ is a continuously differentiable function and $K^{\prime} \subseteq Y^{\prime}$ is a closed convex set, if for a feasible point $x$,

$$
G^{\prime}(x) X+\operatorname{lin}\left(\mathscr{T}_{K^{\prime}}(G(x))\right)=Y^{\prime},
$$

then the constraint nondegeneracy condition holds at $x$. So, for conic optimization problem (1), the constraint nondegeneracy condition at $x^{*}$ has the following form:

$$
\left(\begin{array}{c}
h^{\prime}\left(x^{*}\right) \\
g^{\prime}\left(x^{*}\right)
\end{array}\right) X+\left(\begin{array}{c}
0 \\
\operatorname{lin}\left(\mathscr{T}_{K}\left(g\left(x^{*}\right)\right)\right)
\end{array}\right)=\left(\begin{array}{c}
\mathfrak{R}^{m} \\
Y
\end{array}\right) \text {. }
$$

It follows from [2] that, if a locally optimal solution $x^{*}$ satisfies (46), then $\Lambda\left(x^{*}\right)$ is a singleton.

For a KKT point $\left(x^{*}, y^{*}, z^{*}\right) \in X \times \Re^{m} \times Y$ of problem (1), suppose that $u^{*}:=g\left(x^{*}\right)-z^{*}$ has the spectral decomposition:

$$
u^{*}=\lambda_{1} c_{1}+\cdots+\lambda_{r} c_{r}
$$

satisfying

$$
\lambda_{1} \geq \cdots \geq \lambda_{s}>0=\lambda_{s+1}=\cdots=\lambda_{s_{1}}>\lambda_{s_{1}+1} \geq \cdots \geq \lambda_{r} .
$$

Then,

$$
\begin{aligned}
g\left(x^{*}\right) & =\sum_{j=1}^{s} \lambda_{j} c_{j}, \\
z^{*} & =-\sum_{j=s_{1}+1}^{r} \lambda_{j} c_{j} .
\end{aligned}
$$

According to (23) and (25), we have

$$
\operatorname{lin}\left(\mathscr{T}_{K}\left(g\left(x^{*}\right)\right)\right)=\left\{w \in Y: w_{\beta \beta}=0, w_{\beta \gamma}=0, w_{\gamma \gamma}=0\right\} .
$$

Although the critical cone $\mathscr{C}\left(x^{*}\right)$ of problem (1) has an explicit formula

$$
\begin{aligned}
\mathscr{C}\left(x^{*}\right)= & \left\{d \in X: f^{\prime}\left(x^{*}\right) d \leq 0,\right. \\
& \left.h^{\prime}\left(x^{*}\right) d=0, g^{\prime}\left(x^{*}\right) d \in \mathscr{T}_{K}\left(g\left(x^{*}\right)\right)\right\},
\end{aligned}
$$

aff $\mathscr{C}\left(x^{*}\right)$ is not easy to describe. Instead, we define the following outer approximation set to aff $\mathscr{C}\left(x^{*}\right)$ with respect to $\left(y^{*}, z^{*}\right)$ :

$$
\begin{aligned}
\operatorname{app}\left(y^{*}, z^{*}\right) & =\left\{d \in X: h^{\prime}\left(x^{*}\right) d=0,\left[g^{\prime}\left(x^{*}\right) d\right]_{\beta \gamma}\right. \\
& \left.=0,\left[g^{\prime}\left(x^{*}\right) d\right]_{\gamma \gamma}=0\right\} .
\end{aligned}
$$

It is easy to get that, for any $\left(y^{*}, z^{*}\right) \in \Lambda\left(x^{*}\right)$,

$$
\text { aff } \mathscr{C}\left(x^{*}\right) \subseteq \text { app }\left(y^{*}, z^{*}\right) .
$$

We now introduce a kind of strong second-order sufficient condition for problem (1), which is coincided with the strong second-order sufficient condition in $[8,9]$ when $K$ is a SDP cone and a second-order cone.

Definition 2. Let $x^{*}$ be a feasible point of (1) such that constraint nondegeneracy condition (46) holds at $x^{*}$. We say that the strong second-order sufficient condition holds at $x^{*}$ if

$$
\begin{array}{r}
\left\langle d, \nabla_{x x}^{2} L\left(x^{*}, y^{*}, z^{*}\right) d\right\rangle+\Upsilon_{g\left(x^{*}\right)}\left(z^{*}, g^{\prime}\left(x^{*}\right) d\right)>0, \\
\forall d \in \operatorname{app}\left(y^{*}, z^{*}\right) \backslash\{0\},
\end{array}
$$

where $\left\{\left(y^{*}, z^{*}\right)\right\}=\Lambda\left(x^{*}\right) \subset \Re^{m} \times Y$ and app $\left(y^{*}, z^{*}\right)$ is defined by (52).

The following theorem establishes the relationship between the strong second-order sufficient condition and the nonsingularity of Clarke's Jacobian of the mapping $F$ defined by (44).

Theorem 2. Let $x^{*}$ is a local minimizer of (1). Assume that $\left(x^{*}, y^{*}, z^{*}\right) \in X \times \Re^{m} \times Y$ is a KKT point to (1). If the constraint nondegeneracy condition and the second-order sufficient condition (53) hold at $x^{*}$, then any element in $\partial F\left(x^{*}, y^{*}, z^{*}\right)$ is nonsingular.

Proof. Firstly, we assume that the strong second-order sufficient condition (53) holds at $x^{*}$ with the constraint nondegeneracy condition (46). We shall prove that any $\mathscr{W} \in \partial F\left(x^{*}, y^{*}, z^{*}\right)$ is nonsingular. Let $(\Delta x, \Delta y, \Delta z) \in X \times$ $\mathfrak{R}^{m} \times Y$ satisfying the condition

$$
\mathscr{W}(\Delta x, \Delta y, \Delta z)=0 .
$$

Suppose that $u=g\left(x^{*}\right)-z^{*}$ has the spectral decomposition (47) satisfying (48), then we can write $g\left(x^{*}\right)$ and $z^{*}$ in the form of (49). From Lemma 1, there exists $V \in \partial \Pi_{K}\left(g\left(x^{*}\right)\right)$ such that

$$
\begin{aligned}
& \mathscr{W}(\Delta x, \Delta y, \Delta z) \\
& =\left(\begin{array}{c}
\nabla_{x x}^{2} L\left(x^{*}, y^{*}, z^{*}\right) \Delta x+\nabla h\left(x^{*}\right) \Delta y-\nabla g\left(x^{*}\right) \Delta z, \\
-h^{\prime}\left(x^{*}\right) \Delta x, \\
-g^{\prime}\left(x^{*}\right) \Delta x+V\left(g^{\prime}\left(x^{*}\right) \Delta x-\Delta z\right)
\end{array}\right)=0 .
\end{aligned}
$$

From the third equality in (56), namely, 


$$
g^{\prime}\left(x^{*}\right) \Delta x=V\left(g^{\prime}\left(x^{*}\right) \Delta x-\Delta z\right)
$$

it has

$$
\begin{array}{r}
\sum_{j=s_{1}+1}^{r}\left\langle c_{j}, g^{\prime}\left(x^{*}\right) \Delta x\right\rangle c_{j}=0, \\
\sum_{l=s_{1}+1}^{r} \sum_{j=s+1}^{l-1} \sum_{i=1}^{d}\left\langle v_{j l}^{(i)},\left(g^{\prime}\left(x^{*}\right) \Delta x\right)\right\rangle v_{j l}^{(i)}=0 .
\end{array}
$$

Then, it implies from (52) and (56) that

$$
\begin{aligned}
\Delta x & \in \operatorname{app}\left(y^{*}, z^{*}\right), \\
0 & =\left\langle\Delta x, \nabla_{x x}^{2} L\left(x^{*}, y^{*}, z^{*}\right) \Delta x+\nabla h\left(x^{*}\right) \Delta y-\nabla g\left(x^{*}\right) \Delta z\right\rangle \\
& =\left\langle\Delta x, \nabla_{x x}^{2} L\left(x^{*}, y^{*}, z^{*}\right) \Delta x\right\rangle+\left\langle-\Delta z, g^{\prime}\left(x^{*}\right) \Delta x\right\rangle .
\end{aligned}
$$

Combining with the last equation of (36) and Lemma 4, we obtain

$$
\left\langle\Delta x, \nabla_{x x}^{2} L\left(x^{*}, y^{*}, z^{*}\right) \Delta x\right\rangle+\Upsilon_{g\left(x^{*}\right)}\left(z^{*}, g^{\prime}\left(x^{*}\right) \Delta x\right) \leq 0 .
$$

Comparing with the strong second-order sufficient condition in the strong form (53), we get $\Delta x=0$. Thus, (36) can be expressed in the following form:

$$
\left(\begin{array}{c}
\nabla h\left(x^{*}\right) \Delta y-\nabla g\left(x^{*}\right) \Delta z \\
V(\Delta z)
\end{array}\right)=0 .
$$

From $V(\Delta z)=0$, we have

$$
\begin{aligned}
& (\Delta z)_{\alpha \alpha}=0, \\
& (\Delta z)_{\alpha \beta}=0, \\
& (\Delta z)_{\alpha \gamma}=0 .
\end{aligned}
$$

Then, it follows from the nondegeneracy (46) that there exists a vector $d \in X$ and $w \in \operatorname{lin}\left(\mathscr{T}_{K}\left(g\left(x^{*}\right)\right)\right)$ satisfying

$$
\begin{aligned}
h^{\prime}\left(x^{*}\right) d & =\Delta y, \\
g^{\prime}\left(x^{*}\right) d+w & =-\Delta z .
\end{aligned}
$$

Therefore, we have

$$
\begin{aligned}
\langle\Delta y, \Delta y\rangle+\langle\Delta z, \Delta z\rangle & =\left\langle\Delta y, h^{\prime}\left(x^{*}\right) d\right\rangle-\left\langle\Delta z, g^{\prime}\left(x^{*}\right) d+w\right\rangle \\
& =-\langle w, \Delta z\rangle=0,
\end{aligned}
$$

where the last equation can be obtained by (50) and (62). Thus, $\Delta y=0, \Delta z=0$. Together with $\Delta x=0$, we get that $\mathscr{W}$ is nonsingular. The proof is completed.

\section{Application}

In this section, as an application of the nonsingularity in Theorem 2, we will study the sequential quadratic programming- (SQP-) type method to solving problem (1). Let $\left(x^{k}, y^{k}, z^{k}\right)$ be the current iteration point. The new iteration points $\left(x^{k+1}, y^{k+1}, z^{k+1}\right)$ will be generated by solving the following quadratic problem:
$\left(\mathrm{Sub}_{k}\right)$

$$
\begin{array}{ll}
\min _{\Delta x} \nabla f\left(x^{k}\right)^{T} \Delta x+\frac{1}{2} \Delta x^{T} M_{k} \Delta x, \\
\\
\text { s.t. } \quad h\left(x^{k}\right)+h^{\prime}\left(x^{k}\right) \Delta x=0, \\
& g\left(x^{k}\right)+g^{\prime}\left(x^{k}\right) \Delta x \in K,
\end{array}
$$

where $M_{k}:=M\left(x^{k}, y^{k}, z^{k}\right)$ and $M: X \times \mathfrak{R}^{m} \times Y \longrightarrow X \times X$ is a matrix function satisfying $M\left(x^{*}, y^{*}, z^{*}\right)=$ $\nabla_{x x}^{2} L\left(x^{*}, y^{*}, z^{*}\right)$. This model introduced in [18] to solve the classical nonlinear programming problems is used to solve the nonlinear SDP problems [26, 27] and nonlinear secondorder cone programming problems $[28,29]$, where they may choose the different forms of $M(\cdot, \cdot, \cdot)$.

Theorem 3. Suppose that $\left(x^{*}, y^{*}, z^{*}\right) \in X \times \Re^{m} \times Y$ is a KKT point to (1) and the second-order sufficient condition (53) with constraint nondegeneracy condition holds at $x^{*} x^{*}$. Let the matrix function $M: X \times \mathfrak{R}^{m} \times Y \longrightarrow X \times X$ satisfying $M\left(x^{*}, y^{*}, z^{*}\right)=\nabla_{x x}^{2} L\left(x^{*}, y^{*}, z^{*}\right)$ be semismooth at $\left(x^{*}, y^{*}, z^{*}\right)$. Then, for any $\left(x^{k}, y^{k}, z^{k}\right) \in \mathcal{U}$, a neighborhood of $\left(x^{*}, y^{*}, z^{*}\right)$, (39) has a KKT solution $\left(\Delta x^{k}, y_{\mathrm{QP}}^{k}, z_{\mathrm{QP}}^{k}\right)$ satisfying

$$
\begin{aligned}
& \left\|\Delta x^{k}\right\|+\left\|y_{Q P}^{k}-y^{*}\right\|+\left\|z_{Q P}^{k}-z^{*}\right\| \\
& \quad=O\left(\left\|\left(x^{k}, y^{k}, z^{k}\right)-\left(x^{*}, y^{*}, z^{*}\right)\right\|\right) .
\end{aligned}
$$

Proof. Let $\left(\Delta x, y_{\mathrm{QP}}, z_{\mathrm{QP}}\right)$ be a KKT point of $\left(\mathrm{Sub}_{k}\right)$. Then,

$$
\begin{aligned}
\nabla f\left(x^{k}\right)+z_{k} \Delta x+\nabla h\left(x^{k}\right) y_{Q P}-\nabla g\left(x^{k}\right) z_{Q P} & =0, \\
h\left(x^{k}\right)+h^{\prime}\left(x^{k}\right) \Delta x= & 0, \\
g\left(x^{k}\right)+g^{\prime}\left(x^{k}\right) \Delta x= & \Pi_{K}\left(g\left(x^{k}\right)\right. \\
& \left.+g^{\prime}\left(x^{k}\right) \Delta x-z_{Q P}\right),
\end{aligned}
$$

namely,

$$
\widehat{F}\left(\Delta x, y_{Q P}, z_{Q P}, x^{k}, y^{k}, z^{k}\right)=0
$$

where

$$
\widehat{F}(\zeta, \eta, \xi, x, y, z):=\left(\begin{array}{c}
\nabla f(x)+M(x, y, z) \zeta+\nabla h(x) \eta-\nabla g(x) \xi, \\
h(x)+h^{\prime}(x) \zeta, \\
g(x)+g^{\prime}(x) \zeta-\Pi_{K}\left(g(x)+g^{\prime}(x) \zeta-\xi\right)
\end{array}\right) .
$$

is a function with the variable $(\zeta, \eta, \xi, x, \lambda, \mu) \in$ $X \times \mathfrak{R}^{m} \times Y \times X \times \mathfrak{R}^{m} \times Y$. Let $\tau:=(\zeta, \eta, \xi) \in X \times \mathfrak{R}^{m} \times Y$ and $v:=(x, \lambda, \mu) \in X \times \Re^{m} \times Y$. Let $\tau^{*}=\left(0, y^{*}, z^{*}\right)$ and $v^{*}=\left(x^{*}, y^{*}, z^{*}\right)$. We can easily have the following equation:

$$
\widehat{F}\left(0, y^{*}, z^{*}, x^{*}, y^{*}, z^{*}\right)=\widehat{F}\left(\tau^{*}, v^{*}\right)=0 .
$$


By replacing $\left(x^{k}, M_{k}\right)$ in (65) with, we obtain a new problem expressed as $\left(\mathrm{Sub}_{*}\right)$, and $\left(0, y^{*}, z^{*}\right)$ is an KKT point of $\left(\mathrm{Sub}_{*}\right)$.

Therefore, the strong second-order sufficient condition of $\left(\mathrm{Sub}_{*}\right)$ at $\left(0, y^{*}, z^{*}\right)$ under the constraint nondegeneracy condition (46) has the same expression as condition (53). It is concluded from Theorem 2 that any element $W \in \Pi_{X \times \Re^{m} \times Y} \partial \widehat{F}\left(\tau^{*}, z^{*}\right)$ is nonsingular. Then, there exists a strong semismooth function $\tau(\cdot): \mathscr{U} \longrightarrow X \times \mathfrak{R}^{m} \times Y$ satisfying

$$
\begin{aligned}
\tau\left(\nu^{*}\right) & =\tau^{*}, \\
\widehat{F}(\tau(\nu), \nu) & =0, \quad \forall v \in \mathcal{U},
\end{aligned}
$$

where $\mathscr{U}$ is a neighborhood of $v^{*}$. Denote $\left(\Delta x^{k}, y_{\mathrm{Q} P}^{k}, z_{\mathrm{Q} P}^{k}\right):=\tau\left(\nu^{k}\right)$. Then, $\left(\Delta x^{k}, y_{\mathrm{Q} P}^{k}, z_{\mathrm{Q} P}^{k}\right)$ is a KKT point of $\left(\mathrm{Sub}_{k}\right)$ if $v^{k} \in \mathcal{U}$. By the strong semismoothness of $\tau(\cdot)$,

$$
\left\|\tau\left(\nu^{k}\right)-\tau^{*}\right\|=O\left(\left\|\nu^{k}-v^{*}\right\|\right) .
$$

The proof is completed.

Now we present a local SQP method based on solving SQP-type model $\left(\mathrm{Sub}_{k}\right)$ in each iteration to solve (1).

Next, the primal-dual quadratic convergence of Algorithm 1 is demonstrated by using the semismoothness of $M$ and Theorem 2 .

Theorem 4. Suppose that all the hypotheses of Theorem 3 still hold for Algorithm 1. Then, there exists a neighborhood $\mathcal{U}$ of $\left(x^{*}, y^{*}, z^{*}\right)$ such that, for any $\left(x^{1}, y^{1}, z^{1}\right) \in \mathscr{U}$, the sequence $\left\{\left(x^{k}, y^{k}, z^{k}\right)\right\}$ generated by Algorithm 1 converges to $\left(x^{*}, y^{*}, z^{*}\right)$ quadratically.

Proof. According to Theorem 3, it is easy to verify that the algorithm is well-defined. Denote

$$
\delta_{k}:=\left\|\left(x^{k}, y^{k}, z^{k}\right)-\left(x^{*}, y^{*}, z^{*}\right)\right\| .
$$

We obtain

$$
\begin{aligned}
\Delta x^{k} & =O\left(\delta_{k}\right), \\
y^{k+1}-y^{*} & =O\left(\delta_{k}\right), \\
z^{k+1}-z^{*} & =O\left(\delta_{k}\right),
\end{aligned}
$$

where $\Delta x^{k}$ is a solution to (65), and $y^{k+1}=y_{Q P}^{k}, z^{k+1}=z_{Q P}^{k}$ is the associated multiplier.

As $M\left(x^{*}, y^{*}, z^{*}\right)=\nabla_{x x}^{2} L\left(x^{*}, y^{*}, z^{*}\right)$ and $M$ is semismooth at $\left(x^{*}, y^{*}, z^{*}\right)$,

$$
M_{k}-\nabla_{x x}^{2} L\left(x^{*}, y^{*}, z^{*}\right)=O\left(\left\|\left(x^{k}, y^{k}, z^{k}\right)-\left(x^{*}, y^{*}, z^{*}\right)\right\|\right) .
$$

It follows from the Taylor expansion to (67) at $\left(x^{*}, y^{*}, z^{*}\right), \nabla_{x} L\left(x^{*}, y^{*}, z^{*}\right)=0, x^{k+1}=x^{k}+\Delta x^{k},(74)$, and (75) that

$$
\begin{gathered}
\nabla_{x x}^{2} L\left(x^{*}, y^{*}, z^{*}\right)\left(x^{k+1}-x^{*}\right)+\nabla h\left(x^{*}\right)\left(y^{k+1}-y^{*}\right) \\
-\nabla g\left(x^{*}\right)\left(z^{k+1}-z^{*}\right)=O\left(\delta_{k}^{2}\right), \\
h^{\prime}\left(x^{*}\right)\left(x^{k+1}-x^{*}\right)=O\left(\delta_{k}^{2}\right) .
\end{gathered}
$$

Because of the strongly semismoothness of the projection operator $\Pi_{K}(\cdot)$, we get $V \in \partial \Pi_{K}\left(g\left(x^{*}\right)-z^{*}\right)$ satisfying

$$
\begin{aligned}
& \Pi_{K}\left(g\left(x^{*}\right)-z^{*}\right)=\Pi_{K}\left(g\left(x^{k}\right)+g^{\prime}\left(x^{k}\right) \Delta x^{k}-z_{\mathrm{QP}}^{k}\right) \\
& +V\left(g\left(x^{*}\right)-z^{*}-g\left(x^{k}\right)-g^{\prime}\left(x^{k}\right) \Delta x^{k}+z_{\mathrm{QP}}^{k}\right) \\
& +O\left(\left\|g\left(x^{*}\right)-z^{*}-g\left(x^{k}\right)-g^{\prime}\left(x^{k}\right) \Delta x^{k}+z_{\mathrm{QP}}^{k}\right\|^{2}\right) .
\end{aligned}
$$

Since

$$
\begin{aligned}
g\left(x^{*}\right) & -z^{*}-g\left(x^{k}\right)-g^{\prime}\left(x^{k}\right) \Delta x^{k}+z_{Q P}^{k} \\
= & g\left(x^{*}\right)-g\left(x^{*}\right)-g^{\prime}\left(x^{*}\right)\left(x^{k}-x^{*}\right)+O\left(\left\|x^{k}-x^{*}\right\|^{2}\right) \\
& -g^{\prime}\left(x^{*}\right) \Delta x^{k}+O\left(x^{k}-x^{*}\right) \Delta x^{k}-z^{*}+z^{k+1} g^{\prime}\left(x^{*}\right) \\
& \cdot\left(x^{*}-x^{k+1}\right)-z^{*}+z^{k+1}+O\left(\delta_{k}^{2}\right),
\end{aligned}
$$

it is known that

$$
\begin{gathered}
\Pi_{K}\left(g\left(x^{k}\right)+g^{\prime}\left(x^{k}\right) \Delta x^{k}-z_{\mathrm{Q} P}^{k}\right)=\Pi_{K}\left(g\left(x^{*}\right)-z^{*}\right) \\
-V\left(g^{\prime}\left(x^{*}\right)\left(x^{*}-x^{k+1}\right)-z^{*}+z^{k+1}\right)+O\left(\delta_{k}^{2}\right),
\end{gathered}
$$

which, together with $\Pi_{K}\left(g\left(x^{*}\right)-z^{*}\right)=g\left(x^{*}\right)$ and $\Pi_{K}\left(g\left(x^{k}\right)+g^{\prime}\left(x^{k}\right) \Delta x^{k}-z_{Q P}^{k}\right)=g\left(x^{k}\right)+g^{\prime}\left(x^{k}\right) \Delta x^{k}$, implies

$$
(V-I) g^{\prime}\left(x^{*}\right)\left(x^{k+1}-x^{*}\right)-V\left(z^{k+1}-z^{*}\right)=O\left(\delta_{k}^{2}\right) .
$$

Following from (76), (77), with (81), we obtain

$$
\begin{aligned}
& \left(\begin{array}{ccc}
\nabla_{x x}^{2} L\left(x^{*}, y^{*}, z^{*}\right) & \nabla h\left(x^{*}\right) & -\nabla g\left(x^{*}\right) \\
h^{\prime}\left(x^{*}\right) & 0 & 0 \\
-g^{\prime}\left(x^{*}\right)+V g^{\prime}\left(x^{*}\right) & 0 & -V
\end{array}\right)\left(\begin{array}{c}
x^{k+1}-x^{*} \\
y^{k+1}-y^{*} \\
z^{k+1}-z^{*}
\end{array}\right) \\
& =O\left(\delta_{k}^{2}\right) .
\end{aligned}
$$

Combining (46) and (54) with Theorem 2, we show that

$$
\left\|\left(\begin{array}{c}
x^{k+1}-x^{*} \\
y^{k+1}-y^{*} \\
z^{k+1}-z^{*}
\end{array}\right)\right\|=O\left(\delta_{k}^{2}\right),
$$

which means the quadratic convergence of the sequence $\left\{\left(x^{k}, y^{k}, z^{k}\right)\right\}$. Then, we complete the proof. 


\section{Conclusion}

In this paper, we discuss the nonlinear symmetric conic programming problems. We show that a kind of strong second-order sufficient condition, together with constraint nondegeneracy condition, implies the nonsingularity of Clarke's generalized Jacobian of the mapping $F$ at the KKT point. In the special cases of NLP, SCOP, and SDP, the converses are also true. Then, we demonstrate the local quadratic convergence of the SQP-type method for solving the nonlinear symmetric conic programming problems.

\section{Data Availability}

No data were used to support this study.

\section{Conflicts of Interest}

The authors declare that they have no conflicts of interest.

\section{Acknowledgments}

The research was supported by the National Natural Science Foundation of China under project nos.61573228 and 11501080 and by Natural Science Foundation of Shandong Province of China (no.ZR2017MA034).

\section{References}

[1] J.-S. Pang and L. Qi, "Nonsmooth equations: motivation and algorithms," SIAM Journal on Optimization, vol. 3, no. 3, pp. 443-465, 1993.

[2] J. F. Bonnans and A. Shapiro, Perturbation Analysis of Optimization Problems, Springer, New York, NY, USA, 2000.

[3] F. Facchinei and J. S. Pang, Finite-Dimensional Variational Inequalities and Complementarity Problems, Springer, Berlin, Germany, 2003.

[4] D. Klatte and B. Kummer, "Strong stability in nonlinear programming revisited," The Journal of the Australian Mathematical Society. Series B. Applied Mathematics, vol. 40, no. 3, pp. 336-352, 1999.

[5] S. M. Robinson, "Strongly regular generalized equations," Mathematics of Operations Research, vol. 5, no. 1, pp. 43-62, 1980.

[6] J. F. Bonnans and A. Sulem, "Pseudopower expansion of solutions of generalized equations and constrained optimization problems," Mathematical Programming, vol. 70, pp. 123-148, 1995.

[7] A. L. Dontchev and R. T. Rockafellar, "Characterizations of strong regularity for variational inequalities over polyhedral convex sets," SIAM Journal on Optimization, vol. 6, no. 4, pp. 1087-1105, 1996.

[8] J. F. Bonnans and H. Ramírez, "Perturbation analysis of second-order cone programming problems," Mathematical Programming, vol. 104, no. 2-3, pp. 205-227, 2005.

[9] D. Sun, "The strong second-order sufficient condition and constraint nondegeneracy in nonlinear semidefinite programming and their implications," Mathematics of Operations Research, vol. 31, no. 4, pp. 761-776, 2006.

[10] C. Ding, D. Sun, and L. Zhang, "Characterization of the robust isolated calmness for a class of conic programming problems," SIAM Journal on Optimization, vol. 27, no. 1, pp. 67-90, 2017.
[11] Y. L. Liu and S. H. Pan, Stability study for a clas of conic optimization and generalized equations, Ph.D.Thesis, South China University of Technology, Guangzhou, China, 2018.

[12] B. S. Mordukhovich, J. V. Outrata, and H. Ramírez, "Secondorder variational analysis in conic programming with applications to optimality and stability," SIAM Journal on Optimization, vol. 25, no. 1, pp. 76-101, 2015.

[13] Y. Zhang and L. Zhang, "On the upper lipschitz property of the KKT mapping for nonlinear semidefinite optimization," Operations Research Letters, vol. 44, no. 4, pp. 474-478, 2016.

[14] L. Kong, L. Tunçel, and N. Xiu, "Clarke generalized Jacobian of the projection onto symmetric cones," Set-Valued and Variational Analysis, vol. 17, no. 2, pp. 135-151, 2009.

[15] L. Kong, L. Tunçel, and N. Xiu, "Equivalent conditions for Jacobian nonsingularity in linear symmetric cone programming," Journal of Optimization Theory and Applications, vol. 148, no. 2, pp. 364-389, 2011.

[16] L. Kong, L. Tunçel, and N. Xiu, "Monotonicity of Löwner operators and its applications to symmetric cone complementarity problems," Mathematical Programming, vol. 133, no. 1-2, pp. 327-336, 2012.

[17] Y. Wang, Perturbation analysis of optimization problems over symmetric cones, Ph.D. Thesis, Dalian University of Technology, Dalian, China, 2008.

[18] P. T. Boggs, "Sequential quadratic programming," Acta Numerica, vol. 4, pp. 1-51, 1995.

[19] Z. X. Chan and D. Sun, "Constraint nondegeneracy, strong regularity, and nonsingularity in semidefinite programming," SIAM Journal on Optimization, vol. 19, no. 1, pp. 370-396, 2008.

[20] F. H. Tolle, Optimization and Nonsmooth Analysis, John Wiley \& Sons, Hoboken, NJ, USA, 1983.

[21] B. Kummer, "Lipschitzian inverse functions, directional derivatives, and applications in $C^{1,1}$-optimization," Journal of Optimization Theory and Applications, vol. 70, pp. 559-580, 1991.

[22] F. Meng, D. Sun, and G. Zhao, "Semismoothness of solutions to generalized equations and the Moreau-Yosida regularization," Mathematical Programming, vol. 104, no. 2-3, pp. 561-581, 2005.

[23] J. Faraut and A. Korányi, Analysis on Symmetric Cones, Clarendon Press, Oxford, UK, 1994.

[24] A. Korányi, "Monotone functions on formally real Jordan algebras," Mathematische Annalen, vol. 269, pp. 73-76, 1984.

[25] D. Sun and J. Sun, "Löwner's operator and spectral functions in euclidean Jordan algebras," Mathematics of Operations Research, vol. 33, no. 2, pp. 421-445, 2008.

[26] R. Correa and H. R. Ca, "A global algorithm for nonlinear semidefinite programming," SIAM Journal on Optimization, vol. 15, no. 1, pp. 303-318, 2004.

[27] Y. Wang, S. Zhang, and L. Zhang, "A note on convergence analysis of an SQP-type method for nonlinear semidefinite programming," Journal of Inequalities and Applications, vol. 2008, Article ID 218345, 10 pages, 2008.

[28] H. Kato and M. Fukushima, "An SQP-type algorithm for nonlinear second-order cone programs," Optimization Letters, vol. 1, no. 2, pp. 129-144, 2007.

[29] Y. Wang and L. Zhang, "Properties of equation reformulation of the karush-kuhn-tucker condition for nonlinear second order cone optimization problems," Mathematical Methods of Operations Research, vol. 70, no. 2, pp. 195-218, 2009. 